\title{
Experimental Realization of the Quantum Box Problem
}

\author{
K.J. Resch ${ }^{1}$, J.S. Lundeen ${ }^{2}$, and A.M. Steinberg ${ }^{1,2}$ \\ ${ }^{1}$ Institut für Experimentalphysik, Universität Wien, Boltzmanngasse 5, 1090, Vienna, AUSTRIA \\ ${ }^{2}$ Department of Physics, University of Toronto, \\ 60 St. George Street, Toronto, ON M5S 1 A7 CANADA
}

\begin{abstract}
The three-box problem is a gedankenexperiment designed to elucidate some interesting features of quantum measurement and locality. A particle is prepared in a particular superposition of three boxes, and later found in a different (but nonorthogonal) superposition. It was predicted that appropriate "weak" measurements of particle position in the interval between preparation and post-selection would find the particle in two different places, each with certainty. We verify these predictions in an optical experiment and address the issues of locality and of negative probability.
\end{abstract}

Weak measurements have been controversial ever since the concept was developed by Aharonov, Albert, and Vaidman (AAV) [1]. In contrast to the usual, von Neumann, approach to measurement, weak measurement uses an apparatus whose pointer has a very large quantum mechanical uncertainty when compared with its typical shift. After the system-pointer interaction, the shift in the pointer position is much smaller than its initial uncertainty and almost no information is gained about the quantum system. Nevertheless, after a sufficiently large number of measurements on an ensemble of identically prepared quantum systems, the mean pointer position can be determined to any degree of precision. In such a measurement strategy, one sacrifices knowledge of the value of an observable on any given experimental run to avoid entanglement with the measurement device and the ensuing 'collapse' of the wavefunction. In particular, this makes it possible to contemplate the behavior of a system defined both by state preparation and by a later post-selection, without significant disturbance of the system in the intervening period.

AAV 1] calculated the shift in the pointer of a measurement apparatus that weakly measured an observable $A$ between two strong measurements. The initial strong measurement pre-selects (or prepares) the state, $\left|\psi_{i}\right\rangle$, of the quantum system and the final strong measurement post-selects the quantum state, $\left|\psi_{f}\right\rangle$. In between, consider a von Neumann-style interaction Hamiltonian of the form

$$
\mathcal{H}_{I}=g \hat{A} \hat{P}_{x}
$$

where $\hat{A}$ is the hermitian operator corresponding to an observable $A$ of the quantum system, $g$ is a (real) coupling constant, and $\hat{P}_{x}$ is the momentum operator conjugate to the pointer position $\hat{X}$. In the absence of postselection, the effect of having this measurement interaction on for a time $T$ (assumed short enough that $A$ is constant during the measurement) shifts the pointer position by an amount $\Delta x=K\langle\hat{A}\rangle \equiv g T\langle\hat{A}\rangle$, such that one can infer a value for $A$ by dividing the pointer shift by the interaction strength $K$. The main result of AAV's seminal work [1] is that for sufficiently weak coupling strength $K$ and in the presence of postselection, the inferred value of
$A$ is given by the following expression, which they called the "weak value":

$$
A_{W}=\frac{\left\langle\psi_{f}|\hat{A}| \psi_{i}\right\rangle}{\left\langle\psi_{f} \mid \psi_{i}\right\rangle} .
$$

When the two strong measurements pre- and post-select the same state, $A_{W}$ reduces to the usual quantum mechanical expectation value for the operator $\hat{A}$. However when the pre- and post-selected states differ, weak values can take on surprising values that are not constrained to lie within the eigenvalue spectrum of the operator or even to be real numbers. The surprising character of weak values has led to skepticism about whether they should be considered proper measurements $[2,[3,4]$. In spite of their controversial nature, a weak measurement has been experimentally performed by Ritchie et al. [5]; in addition, they have been useful in correctly describing or predicting surprising experimental outcomes 1, 6, 7, 8, 9, 10, 11].

The "Quantum Box Problem" was developed by Aharonov and Vaidman [12, 13]; it is a deceptively simple thought experiment that elucidates some of the odd behavior which may result from studying post-selected systems. In the 3-box version, a particle is prepared (preselected) in an equally weighted superposition of being in one of three orthogonal quantum "boxes", $\mathrm{A}, \mathrm{B}$, and $\mathrm{C}$, i.e., $\left|\psi_{i}\right\rangle=\frac{1}{\sqrt{3}}|A\rangle+\frac{1}{\sqrt{3}}|B\rangle+\frac{1}{\sqrt{3}}|C\rangle$ and post-selected in the final state $\left|\psi_{f}\right\rangle=\frac{1}{\sqrt{3}}|A\rangle+\frac{1}{\sqrt{3}}|B\rangle-\frac{1}{\sqrt{3}}|C\rangle$. The weak value for the particle being in box $\mathrm{A}$ can be calculated by inserting the projection operator $|A\rangle\langle A|$ in Eq. 2 i.e., $A_{W}=\left\langle\psi_{f} \mid A\right\rangle\left\langle A \mid \psi_{i}\right\rangle /\left\langle\psi_{f} \mid \psi_{i}\right\rangle=+1$. In this case, the pointer shift is the same as in the case when the particle was definitely in box A. We call this quantity the "weak probability for the particle in box A" and use the shorthand $P_{A W}$, where $A$ represents the rail. This weak probability is already strange since a strong measurement would find the particle in box A only $|1 / \sqrt{3}|^{2}=1 / 3$ of the time. Through a similar calculation, the weak probability for the particle in box $\mathrm{B}, P_{B W}$, is also found be be +1 . Like normal probabilities, the sum of all weak probabilities is +1 (because the sum of all orthogonal projectors is the identity); therefore, if one performs a weak measurement of the particle in box $\mathrm{C}$ one finds the 
weak probability, $P_{C W}=\left\langle\psi_{f} \mid C\right\rangle\left\langle C \mid \psi_{i}\right\rangle /\left\langle\psi_{f} \mid \psi_{i}\right\rangle=-1$. Such a result is very strange since it lies outside the range of eigenvalues for projection operators and normal probabilities - i.e., it does not lie between 0 and 1 . Nevertheless, for a large enough ensemble of identically prepared and post-selected states, this result properly predicts the outcome of our experiment. Note that while apparently similar to the situation for quasi-probability distributions such as the Wigner function, this case is different, referring instead to a directly observable measurement outcome. There are also other situations in which it has been suggested that negative probabilities might be useful for resolving locality "paradoxes" 14]. For a review of such extensions to probability theory, see [15].

We use a linear optical interferometer to implement the quantum box problem (Fig. 1). This interferometer is similar to the Mach-Zehnder interferometer except that it has three optical paths (or rails) instead of two; we label these rails A, B, and C. A photon can be prepared in any superposition of these three rails by proper selection of the characteristics of beamsplitters BS1 and BS2. Similarly, any coherent superposition can be postselected by controlling the characteristics of BS3, BS4, and the optical path lengths (relative phases). We use the transverse displacement of the photon as our measurement pointer. Measurements are carried out by tilting one of the glass plates (GP A, B, or C); this results in a controllable transverse shift of the beam in one of the rails. For example, a glass plate in rail A displacing the beam along $X$ can be thought of in terms of an effective interaction Hamiltonian of the form $g|A\rangle\langle A| \hat{P}_{X}$. The measurement of the mean spatial shift of an ensemble of photons can be viewed as a measurement of the probability for each photon to have been in rail A. If the transverse shift is much less than the beam size (the uncertainty in the position of the individual photons), this is a weak measurement. Shifting only one rail at a time, we measure the size of that shift while keeping the other two rails blocked. This characterizes the strength $K_{A}$ of the system-pointer interaction. We then combine all three beams with the correct relative phases for proper post-selection and again measure the shift $\Delta x$. The ratio of the shift in the post-selected state to that of the single rail constitutes our weak probability for that rail $A_{W}=\Delta x / K_{A}$. A negative weak probability is realized when the shift in the post-selected state is in the opposite direction to the shift on the individual rail.

For the experiment, light from a $780 \mathrm{~nm}, 30 \mathrm{~mW}$, diode laser is spatially filtered to yield a collimated TEM $_{00}$ gaussian beam with a waist of $380 \mu \mathrm{m}$; this amounts to a large ensemble of identically-prepared photons. To create the appropriate superposition, the transmissivity and reflectivity of each beam-splitter is adjusted by using two half-wave plates $(\lambda / 2)$ prior to polarizing beam-splitters (PBS). The third half-wave plate is present to rotate the polarization in rail $\mathrm{C}$ from horizontal to vertical so that all three rails have the same polarization. Glass plates with thicknesses $10 \mathrm{~mm}, 6.5 \mathrm{~mm}$, and $10 \mathrm{~mm}$ are situated in paths $\mathrm{A}, \mathrm{B}$, and $\mathrm{C}$ respectively. The plate in rail $\mathrm{A}$ displaces the beam in the vertical direction (out of the plane of the inteferometer) while the plates in rails $\mathrm{B}$ and $\mathrm{C}$ displace the beam in the horizontal direction (in the plane of the interferometer). In addition to these glass plates, 1-mm-thick microscope slides are located in rails $\mathrm{A}$ and $\mathrm{C}$. These plates can also be finely tilted to set the relative phases, $\phi_{A}$ and $\phi_{C}$, between the light in these arms with that in rail $\mathrm{B}$ without significantly displacing the beam. Rails A and B are recombined coherently at beam-splitter (BS) 3. One of the outputs from BS3 is then recombined with the light from rail $\mathrm{C}$ at $\mathrm{BS} 4$. The phases are chosen such that the light in the right-going output of BS4 is our desired post-selected state. This beam is magnified by a factor of 3 on a screen behind which a CMOS camera (Logitech Quickcam) captures beam images. The second output of BS4 terminates at a photodiode that is used to set the relative phases of the three arms of the interferometer.

The quantum box problem can be generalized for an arbitrary pre-selected state $\left|\varphi_{i}\right\rangle=a_{i}|A\rangle+b_{i}|B\rangle+c_{i}|C\rangle$ and post-selected state $\left|\varphi_{f}\right\rangle=a_{f}|A\rangle+b_{f}|B\rangle-c_{f}|C\rangle$. If the coefficients are real numbers, we obtain the same weak values discussed earlier for rails $\mathrm{A}, \mathrm{B}$, and $\mathrm{C}$ if $a_{i} a_{f}=$ $b_{i} b_{f}=c_{i} c_{f}$, albeit with a lower overlap between our preand post-selected states. These requirements can be converted into experimental parameters in the following way. Given reflection and transmission amplitudes $r_{i} \exp \left(i \phi_{r i}\right)$ and $t_{i} \exp \left(i \phi_{t i}\right)$ for $\mathrm{BS} i$, the 3-path interferometer preselects the state $\left|\varphi_{i}\right\rangle=r_{1}|A\rangle+t_{1} r_{2}|B\rangle+t_{1} t_{2}|C\rangle-$ the phases are compensated for by optical path lengths. For the proper phase settings, the interferometer postselects the state $\left|\varphi_{f}\right\rangle=t_{3} r_{4}|A\rangle+r_{3} r_{4}|B\rangle-t_{4}|C\rangle$. To satisfy the condition $a_{i} a_{f}=b_{i} b_{f}=c_{i} c_{f}$, we require $r_{1} t_{3} r_{4}=t_{1} r_{2} r_{3} r_{4}=t_{1} t_{2} t_{4}$. This is the condition that each path contributes the same intensity in the camera output port. In our experiment, the final beamsplitters are both 50/50, and therefore our post-selected state is approximately $\left|\varphi_{f}\right\rangle=\frac{1}{2}|A\rangle+\frac{1}{2}|B\rangle-\frac{1}{\sqrt{2}}|C\rangle$. The proper pre-selected state to obtain the desired weak values is $\left|\varphi_{i}\right\rangle=\sqrt{\frac{2}{5}}|A\rangle+\sqrt{\frac{2}{5}}|B\rangle+\sqrt{\frac{1}{5}}|C\rangle$. We note that this reduces the overlap of the initial and final states from $1 / 3$ for the original states to $\sqrt{1 / 10}$.

The data for this experiment were taken in two parts. In both parts, we began by balancing the intensities in the camera arm from the three paths and aligned the beams in the interferometers such that they overlapped to better than $1 / 10$ of their rms widths. All of the beams in the interferometer were vertically-polarized. Pairwise, beams $\mathrm{A} \& \mathrm{~B}$ and $\mathrm{B} \& \mathrm{C}$ had interference fringe visibilities of about $95 \%$. In the first part of the experiment we performed only single weak measurements on each of the 3 rails. A single glass plate (GP) in one of the arms was tilted to displace the beam in that path. The phases in the interferometers were set using the microscope slides so that paths A and B interfere constructively and paths $\mathrm{B}$ and $\mathrm{C}$ interfere destructively in the 
camera output. Images of each individual beam (in the absence of interference) and the properly post-selected beam were recorded using the camera. In Fig. 2, horizontal profiles are shown, with a particular displacement of rail $\mathrm{C}$, for the beams in rails $\mathrm{A}$ (thin solid line), $\mathrm{B}$ (thin dashed line), and $\mathrm{C}$ (thick dashed line) alone, and also for the post-selected state (thick solid line). Beams A and $B$ have the same average position to within less than 1 pixel, or about $1 / 20$ of the rms width. Beam $\mathrm{C}$ has been displaced from this centre position by -11.1 pixels or $-0.69 \mathrm{rms}$ widths. This is the coupling strength $K_{C}$. The post-selected state beam profile is displaced by +7.0 pixels or $+0.44 \mathrm{rms}$ widths in the opposite direction of rail $\mathrm{C}$; in other words, the observed value $\Delta x / K_{C}=-0.64$. This differs from the expected value $P_{C W}=-1$ because the displacement is already approaching the transition from the weak to the strong measurement regime.

We have summarized our results for post-selected measurements of $P_{A}, P_{B}$, and $P_{C}$ for a variety of coupling strengths in Fig. 3. The observed displacements $\Delta x$ for measurements of $P_{A}$ and $P_{B}$ are shown as open circles and solid triangles respectively, as a function of $K_{\{A, B\}}$. The theoretical prediction for these two rails is a straight line with a slope of 1 and a y-intercept of 0 . The slope of 1 indicates a shift as large as if all the photons had traversed the shifted rail, that is, $P_{A W}=P_{B W}=1$. Note that this slope of 1 persists even into the strongmeasurement regime, $K_{\{A, B\}}>1 \mathrm{rms}$ width. This can be understood from the following argument. If we perform a weak measurement on rail A, then in our postselection output the amplitudes from rails $\mathrm{B}$ and $\mathrm{C}$ interfere perfectly destructively and we are left only with the field from rail A. Therefore, whatever displacement rail A has, so will the post-selected state 13 . . Of course a similar argument can be constructed for rail B. The data for rail $\mathrm{C}$ has different behaviour and no such argument applies. The experimental data is shown as solid circles and the theoretical prediction as a solid line. Near zero displacement of the rail, the displacement of the post-selected state is in the opposite direction as the displacement of rail C. The weak probabilities are given by the slopes of the curves from the plot in Fig 3 near the origin. The weak probabilities are consistent with the predictions of $+1,+1$, and -1 for $P_{A W}, P_{B W}$, and $P_{C W}$ respectively. For larger displacements in rail $\mathrm{C}$, the weakness criterion is not satisfied, and the observed shifts eventually approach a strong-measurement limit of $1 / 5$ [16].

We have shown that the weak probability to find a particle in rail $A$ is +1 , as is the the weak probability to find the particle in rail $B$. This begs the question, what is the weak probability to find the particle in both arms? It is well-known that a single particle cannot trigger two different spacelike separated detectors; in fact one might think of that as the defining quality of a particle - it must be either here or there but never both. Therefore, one would never expect to find a single photon in both rails $\mathrm{A}$ and $\mathrm{B}$ via projective measurements. It has been suggested, however, that this is a limitation of strong measurements; if a particle is prepared in a suitably delocalized state, might it be possible to find that particle in two different places at the same time if the presence of the particle is probed weakly [17]?

For a state involving only one particle, we can use the operator $|A\rangle\langle A \mid B\rangle\langle B|$ to find the joint weak probability for the particle to be in rails $\mathrm{A}$ and $\mathrm{B}$ simultaneously. However, because $|A\rangle$ and $|B\rangle$ represent orthogonal states, this operator is identically zero. Therefore the weak probability to find the particle in both rails is expected to be zero even when the single weak probabilities (i.e., $P_{A W}$ and $P_{B W}$ ) are both equal to 1 . This seeming violation of standard rules of probability can be seen to be related to the relaxation of the non-negativity axiom of probability theory 8, 18].

In the second part of the experiment, we performed two different simultaneous measurements on the photons using two different degrees of freedom at the same time. In addition to the transverse beam displacement in rail $\mathrm{B}$, we used the waveplate in rail $\mathrm{C}$ (see Fig. 1) just before $\mathrm{BS} 4$ to rotate the polarization of the light by a small amount. We prepared the same initial state as before but use a different final state, $\left|\psi_{f 2}\right\rangle=-\frac{1}{2}|A\rangle+\frac{1}{2}|B\rangle+$ $\frac{1}{\sqrt{2}}|C\rangle$. Such a final state swaps the roles of rails $\mathrm{A}$ and $\mathrm{C}$ so that $P_{A W}=-1$ and $P_{C W}=+1$. The reason for doing so was purely technical. Our final beamsplitters were $50 / 50$ for vertically-polarized light but for horizontallypolarized light they had greater than $90 \%$ transmission. We ensured that rail $\mathrm{C}$, where the polarization rotation occurs, was transmitted rather than reflected, so that the observable polarization shift at the camera would not be negligibly small. We rotated the waveplate in rail $\mathrm{C}$ by a small amount that resulted in a $K_{C}=9.6^{\circ}$ rotation in the polarization of the light after the final beamsplitter when rails $A$ and $B$ were blocked.

We measured the displacement, $\Delta x$, of the postselected state as a function of the rail-B coupling strength $K_{B}$. The results, $\Delta x / K_{B}$, are the inferred probabilities of the photon to be in rail B and are shown in Fig. 4., both in the case of no final polarizer (solid circles, solid line theory) and in the case of a final polarizer oriented to block vertically-polarized light (open circles, long-dashed line theory). Recall that all of the beams in the interferometer initially had vertically-polarized light. When the polarizer was inserted to block vertically-polarized light we measured the beam displacement of only the light that had its polarization rotated, i.e., only photons which had traversed rail $\mathrm{C}$. We also show the inferred probability of the photon to be in rail $\mathrm{C}$ - given by the polarization rotation of the final state divided by $K_{C}=9.6^{\circ}$ (open triangles, dotted line theory). The low data point for the $P_{B}$ was most likely due to a mode-hop-induced phase fluctuation, and the slight disagreement between the theory and experimental values for $P_{C}$ is from imperfect interference. These data show, that for displacements of rail $B$ in the weak regime (less than $0.5 \mathrm{rms}$ widths), the entire distribution of the light is both displaced and 
polarization rotated as if the photons really experience both weak measurements. However, by blocking all of the vertically-polarized light, we clearly see that the distribution is not shifted at all. Therefore we can say that those particles that were definitely in rail $\mathrm{C}$ were not even weakly in rail B.

We have implemented the quantum box problem in a 3 -rail interferometer and verified some of the important predictions about the weak measurements one can perform in this system. Specifically, we have observed weak probabilities of +1 for rails $A$ and $B$ and a weak probability of -1 for rail $\mathrm{C}$. We have also studied the transition out of the weak-measurement regime for rail $\mathrm{C}$, when the interaction shifts the measurement pointer by more than about $0.5 \mathrm{rms}$ widths. In addition, we performed two simultaneous measurements on two of the rails using polarization and transverse displacement as pointers. For small displacements, we found that the entire beam was shifted and polarization rotated. However, when only the polarization-rotated light was studied, its transverse displacement was zero. This shows the (one-sided) anticorrelation between a strong measurement of the particle in one rail and a weak measurement of it in another. This leaves open the question of what one would observe by making joint weak measurements on two different rails. In another work, we have proposed an experimentally feasible method for performing such measurements [19], and an ongoing experiment aims to carry them out for the 3-box problem. These types of conceptually simple experiments yield insight into the sometimes shocking behavior of post-selected subensembles in quantum mechanics, and into the power and the limitations of weak measurements.

We would like to thank Morgan Mitchell, Jeff Tollaksen, and Jeeva Anandan for helpful advice and discussions. We gratefully acknowledge financial support from Photonics Research Ontario and NSERC.
[1] Y. Aharonov, D.Z. Albert, and L. Vaidman, Phys. Rev. Lett., 60, 1351 (1988).

[2] A.J. Leggett, Phys. Rev. Lett., 62, 2325 (1989).

[3] A. Peres, Phys. Rev. Lett., 62, 2326 (1989).

[4] Y. Aharonov and L. Vaidman, Phys. Rev. Lett., 62, 2327 (1989).

[5] N.W.M. Ritchie, J.G. Story, and R.G. Hulet, Phys. Rev. Lett., 66, 1107 (1991).

[6] I.M. Duck, P.M. Stevenson, and E.C.G. Sudarshan, Phys. Rev. D, 40, 2112 (1989).

[7] A.M. Steinberg, Phys. Rev. Lett., 74, 2405 (1995).

[8] Y. Aharonov, A. Botero, S. Popescu, B. Reznik, and J. Tollaksen, Phys. Lett. A, 301, 130 (2001); L. Hardy, Phys. Rev. Lett., 68, 2981 (1992).

[9] H.M. Wiseman, Phys. Lett. A, 311, 285 (2003).

[10] K. Mølmer, Phys. Lett. A, 292, 151 (2001).

[11] G.T. Foster, L.A. Orozco, H.M. Castro-Beltran, and H.J. Carmichael, Phys. Rev. Lett., 85, 3149 (2000); H. M. Wiseman, Phys. Rev. A, 65, 032111 (2002).

[12] Y. Aharonov and L. Vaidman, J. Phys. A: Math. Gen., 24, 2315 (1991).

[13] L. Vaidman, Foundations of Physics, 26, 895 (1996).

[14] R.P. Feynman, International Journal of Theoretical Physics, 21, 467 (1982); M.O. Scully, H. Walther, W. Schleich, Phys. Rev. A, 49, 1562 (1994).

[15] W. Muckenheim, Phys. Rep., 133, 339 (1983).

[16] Y. Aharonov, P.G. Bergmann, and J.L. Lebowitz, Phys. Rev., 134, B1410 (1964).

[17] A.M. Steinberg, "Can a falling tree make a noise in two forests at the same time?", in Causality and Locality in Modern Physics, S. Jeffers, G. Hunter, and J.-P. Vigier, eds., Kluwer Academic Publishers (Dordrecht: 1997), p. 431; A. M. Steinberg, S. Myrskog, Han Seb Moon, Hyun Ah Kim, Jalani Fox, Jung Bog Kim, Ann. Phys. (Leipzig), 7, 593 (1998).

[18] A.M. Steinberg, to appear in SCIENCE AND ULTIMATE REALITY: Quantum Theory, Cosmology and Complexity, eds. John D. Barrow, Paul C.W. Davies, and Charles L. Harper, Jr., Cambridge University Press,
2003; quant-ph/0302003

[19] K.J. Resch and A.M. Steinberg, "Extracting joint weak values with local, single-particle measurements", submitted (2003). 
Figure 1. The 3-rail Mach-Zehnder-style interferometer. The TEM To $_{0}$ mode of a diode laser is filtered spatially using a pinhole (PH), two lenses and an iris. Light is placed in the proper coherent superposition of each of the rails labelled $A$, B, and $\mathbf{C}$ using half-wave plates $(\lambda / 2)$ and polarizing beam-splitters (PBSs). Glass plates (GPs) in each arm are used to displace each beam transverse to its direction of propagation, and microscope slides (MS) are used to finely adjust the phases in arms $\mathrm{A}$ and $\mathrm{C}$. The three modes are recombined at two 50/50 beamsplitters (BS3 and BS4). The beam shines on a screen and a CMOS camera behind that screen captures its image. A photodiode (PD) in the second port of BS4 is used to set the relative phases light in the different rails.

Figure 2. Sample experimental data when only rail $\mathbf{C}$ is displaced. We show experimental data for individual horizontal beam profiles from Rails A (thin solid line), B(thin dashed line), and C (thick dashed line) when a transverse, horizontal displacement of beam $\mathrm{C}$ is applied. The beam profile of the post-selected beam is also shown (thick solid line). The beam profile from rail $\mathbf{C}$ is displaced by -11.1 pixels, or $-0.69 \mathrm{rms}$ widths, which constitutes a measure of the coupling strength $K_{C}$. The corresponding shift $\Delta x$ in the post-selected beam is +7.0 pixels, or +0.44 rms widths, implying a probability of $P_{C}=-0.64$. (This is smaller than the expected $P_{C W}=-1$ because in this image, the coupling strength is too large for a true weak measurement. This image is shown for clarity, while Figure 3 summarizes the data for weaker couplings as well, where the agreement with weak-measurement theory is good.)

Figure 3. Experimental data for individual rail displacements. The displacement of the post- selected state is shown as a function of the displacement of each individual rail. The data for rails $\mathrm{A}, \mathrm{B}$, and $\mathrm{C}$ are shown as open circles, solid triangles, and solid circles respectively. Theory (with no adjustable parameters) is shown as a dashed line for rails $A$ and $B$ and as a solid line for rail C. The transition for the displacement of rail $\mathrm{C}$ from the weak measurement regime to the strong measurement regime is clearly visible at a beam displacement of about $\pm 1 \mathrm{rms}$ width. No such transition was observed or predicted for the other two rails.

Figure 4. The weak probabilities for the photon to be in rail $B$ and rail $C$ measured in the same experimental setup using two different pointers. The weak probability for the photon to be in rail $\mathrm{C}$ was measured using a small polarization rotation, and the probability for rail $\mathrm{B}$ was measured with a transverse beam displacement. Both are shown as functions of the displacement of rail $B$, with the polarization rotation being kept fixed. The resulting polarization rotations and displacements in the post-selected states were converted into weak probabilities by dividing by the relevant interaction strengths. The resulting weak probabilities for rails $\mathrm{B}$ and $\mathrm{C}$ are shown as solid circles and open triangles. For very weak measurements, the entire post-selected state is polarization rotated and displaced, by amounts which would imply that each photon was both in rail $\mathrm{C}$ and rail $\mathrm{B}$ with certainty. However, when a polarizer is inserted to block vertically-polarized light (such that we only detect the photons that have been polarization-rotated in rail $\mathrm{C}$ ) we find the weak probability for the photon to have been in rail $\mathrm{B}$ to be zero (open circles, dashed line). Thus, those photons that were definitely in rail $\mathrm{C}$ were definitely not in rail $\mathrm{B}$. 


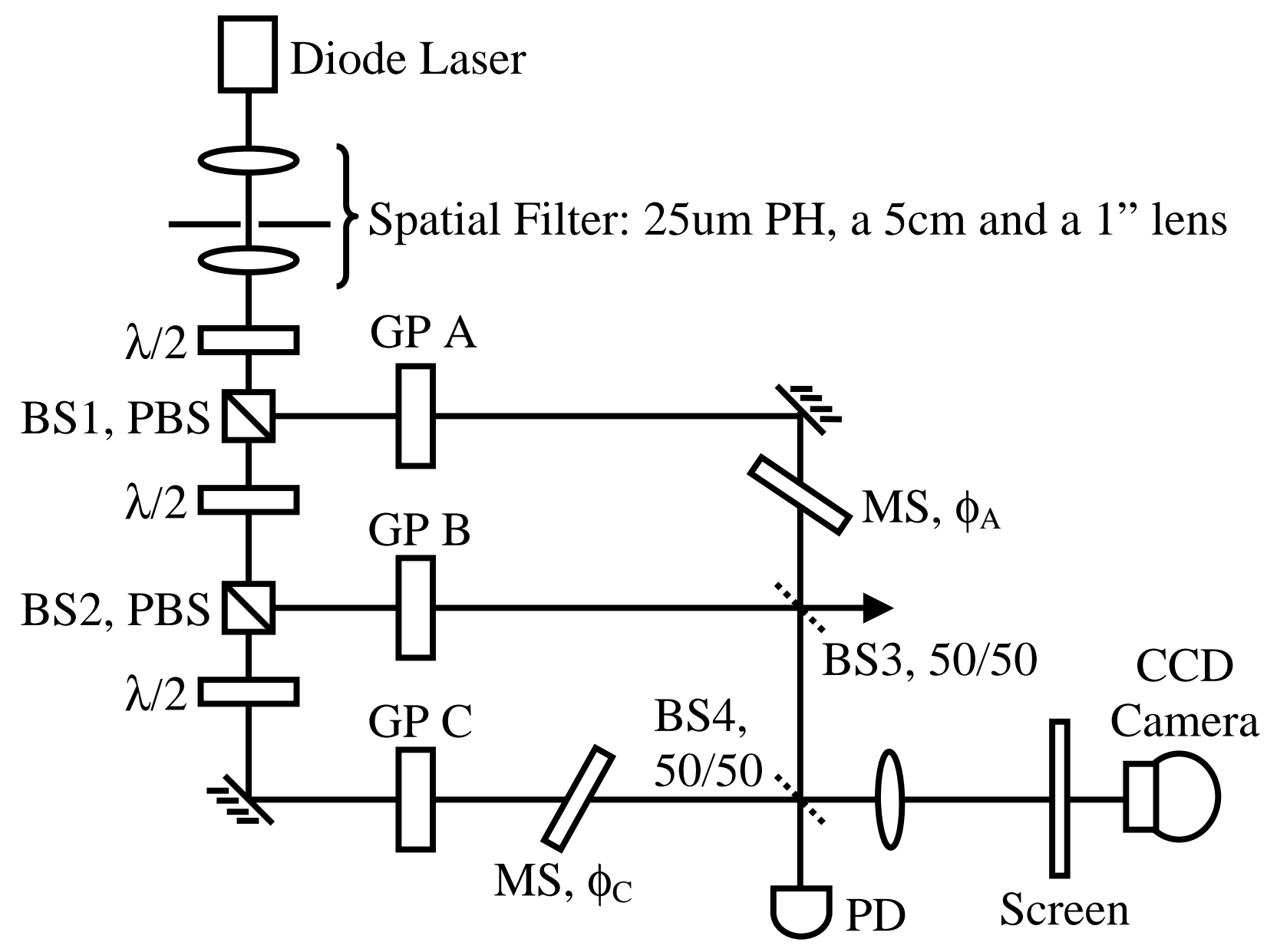




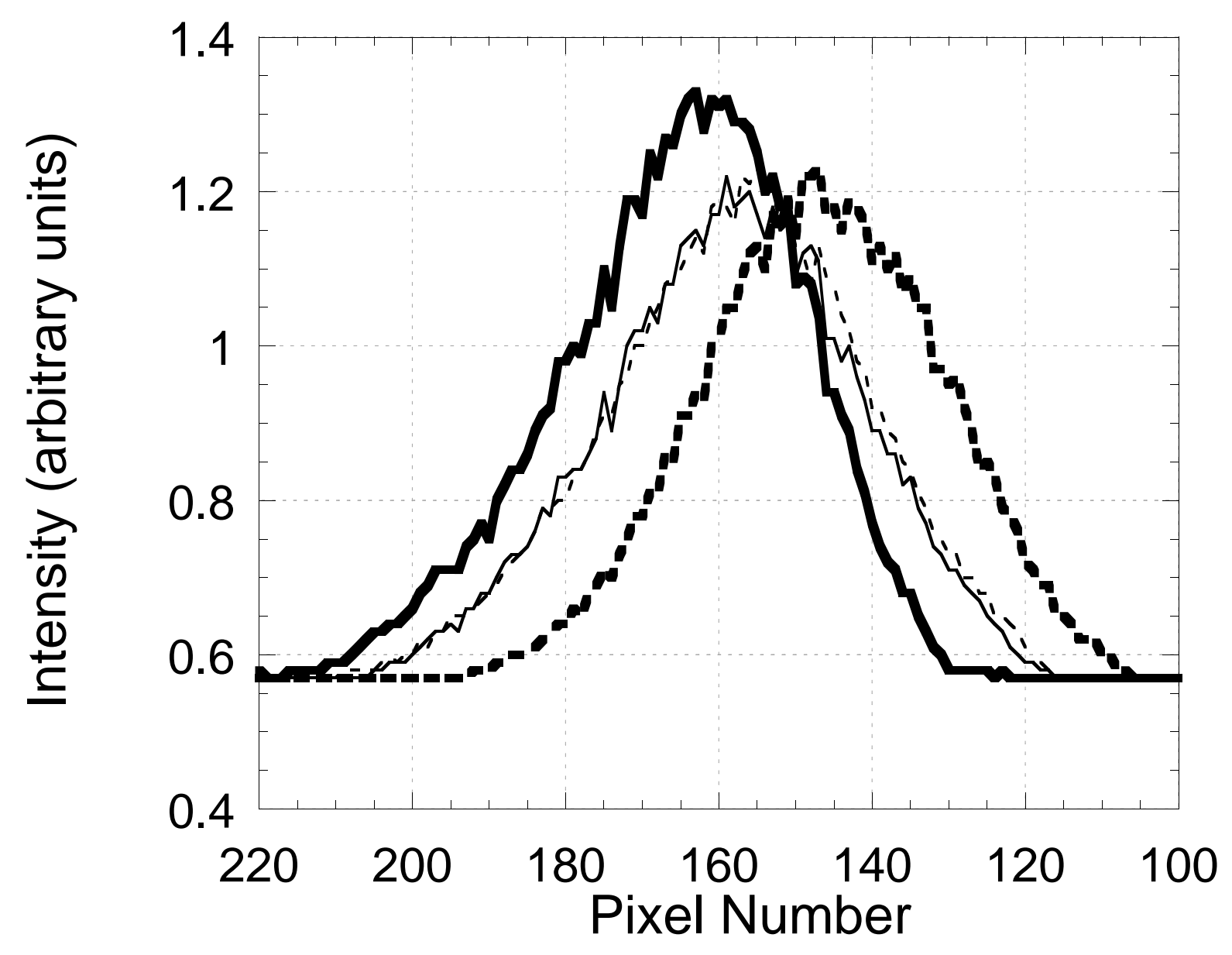




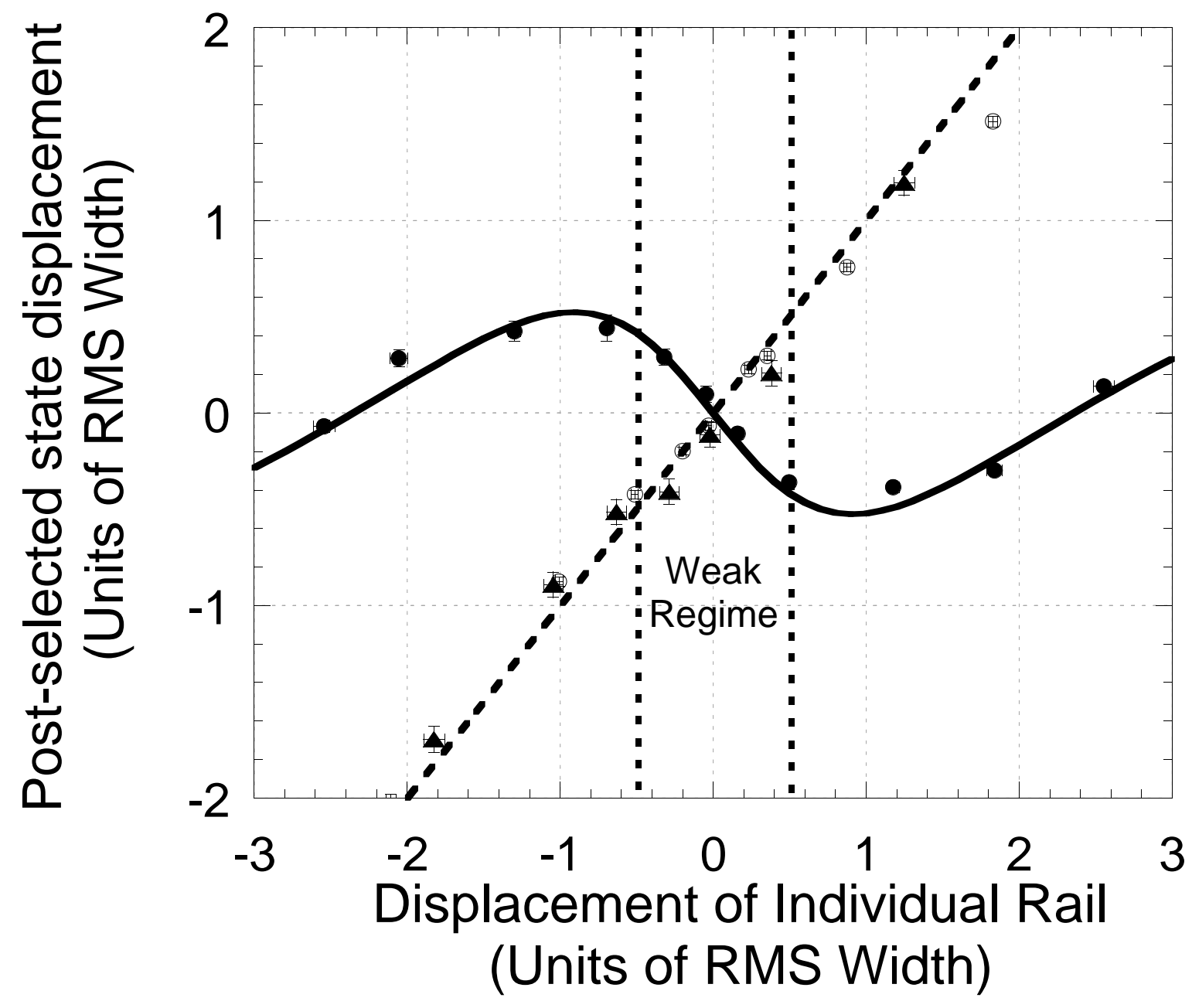




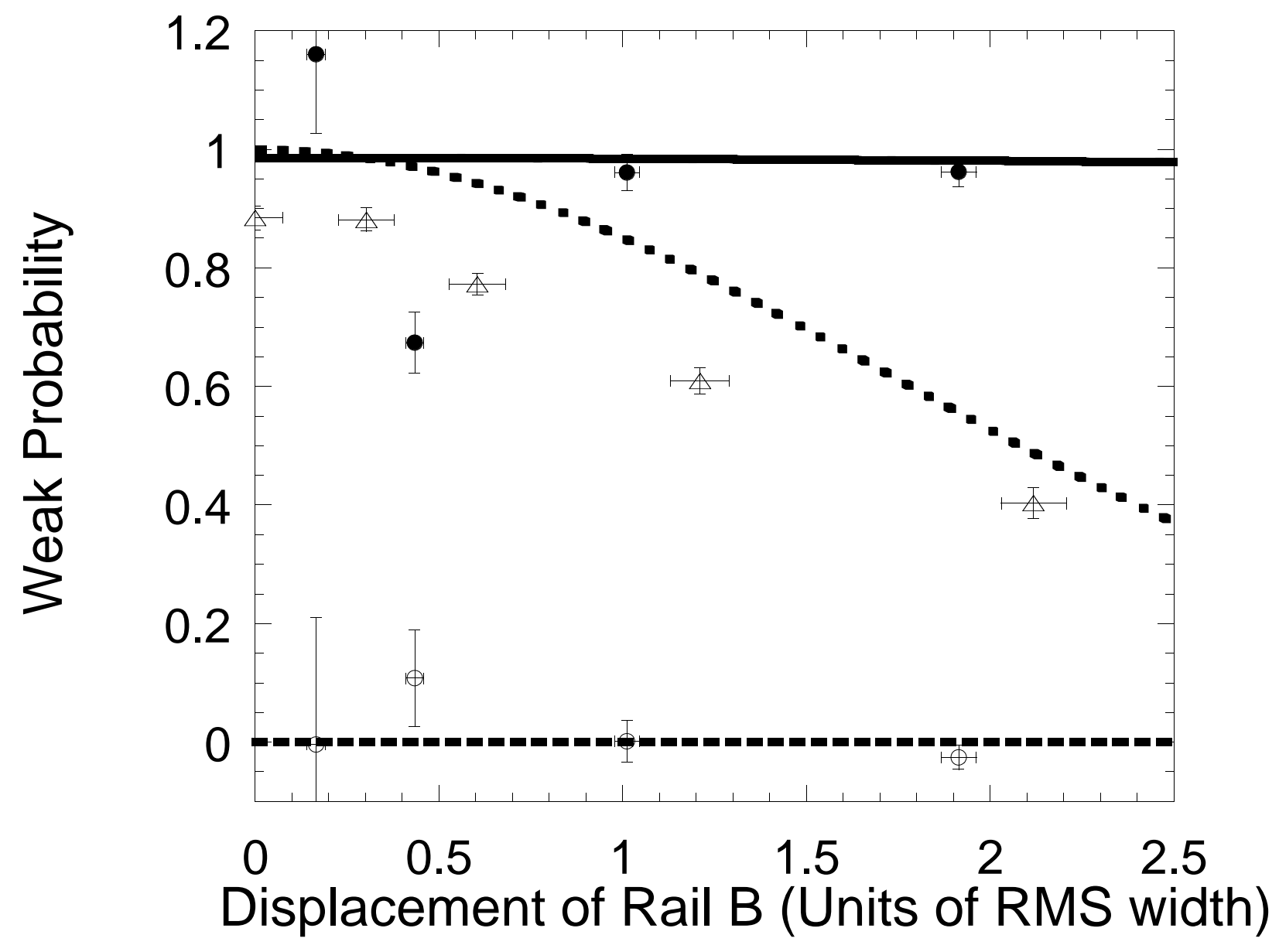

